\title{
Frontal fibrosing alopecia: a disease that remains enigmatic
}

\author{
Anna Lis-Święty, Ligia Brzezińska-Wcisło \\ Department of Dermatology, School of Medicine, Medical University of Silesia, Katowice, Poland \\ Adv Dermatol Allergol 2020; XXXVII (4): 482-489 \\ DOI: https://doi.org/10.5114/ada.2020.98241
}

\begin{abstract}
Frontal fibrosing alopecia (FFA) is recognized to represent a generalized process of inflammatory scarring alopecia. Apart from the classic form affecting the frontal hairline, there are a range of disease manifestations involving loss of eyebrows and of eyelashes, loss of peripheral body hair, fibrosing alopecia in a pattern distribution, facial and extrafacial skin, mucous membrane, and nail involvement. Classic linear, diffuse "zigzag", pseudo "fringe sign", androgenetic alopecia-like, cockade-like, ophiasis-like and incomplete patterns are distinguished. The aetiology of FFA remains obscure, but a number of pathogenetic hypotheses and treatments to halt disease progression have been proposed.
\end{abstract}

Key words: scarring alopecia, etiopathogenesis, diagnostics, therapy.

\section{Introduction}

Frontal fibrosing alopecia (FFA) is a lymphocytic primary scarring alopecia characterized by slow, progressive recession of the frontotemporal hairline with complete loss of hair and follicular openings, most commonly affecting postmenopausal females. Based on histological findings, FFA is thought to be a possible clinical variant of lichen planopilaris (LPP). The precise incidence and prevalence of FFA remains unclear. In the past 20 years, however, there has been an enormous increase in the incidence of FFA cases worldwide, predominantly affecting individuals of higher socio-economic status [1]. Even though the initial cases only comprised Caucasian postmenopausal women, there are now reports of patients of different ethnic backgrounds and case series of FFA affecting premenopausal women ( $15 \%$ of cases), men (3-5\% of cases) and even children (three paediatric cases reported) [1-3]. Determining the precise age of onset is difficult because of the slowly progressive nature of the disease. According to a large meta-analysis, the mean age at disease onset was 55.5 years, the diagnosis was frequently made years later, with most patients being older than 60 years at the time of diagnosis [1]. Men with FFA present with the disease at a younger age than women [4]. However, it is possible that many female are diagnosed post-menopause although the actual onset of FFA may have occurred before menopause. It should be emphasized that the association between FFA and androgenetic alopecia (AGA) can lead to the underdiagnosis of FFA in males with alopecia.

\section{Etiopathogenesis}

The aetiology of FFA is unknown. Genetic, hormonal, autoimmune, inflammatory and environmental factors are suggested to contribute to the pathophysiology. Up to $8 \%$ of FFA cases are thought to be familial [5]. Three monozygotic twins have been identified with FFA [5]. At present, the genetic background of FFA remains poorly characterized. An androgen-dependent aetiology has been proposed based on the predominance of postmenopausal women, frequent coexistence with AGA, and the clinical improvement seen with anti-androgens. There is a higher incidence of FFA in women with early menopause/hysterectomy/oophorectomy [5]. However, no marked hormonal abnormalities were detected by the sex hormone analyses in premenopausal women with FFA [6]. In contrast, low androgen was the most prominent dysfunction type in postmenopausal women with FFA while LPP was associated with androgen excess [7]. Interestingly, Lobato-Berezo et al. report FFA in a male patient after starting a hormonal therapy with antiandrogens (bicalutamide and goserelin) for prostate cancer [8]. Hypothyroidism was present in up to $30 \%$ of FFA cases in several studies, suggesting an autoimmune mechanism in the pathogenesis of the disease. According to Katoulis et al., the melanocyte of the hair follicle might be an antigenic target in FFA [9]. Histological analysis showed lower melanocyte counts in FFA patients were associated with clinical observations of skin hypopigmentation [10]. Melanocyte-derived autoantigens might play a role in the

Address for correspondence: Anna Lis-Święty MD, PhD, Department of Dermatology, School of Medicine, Medical University of Silesia, 20/24 Francuska St, 40-027 Katowice, Poland, phone: +48 602720 948, e-mail: annadlis@neostrada.pl Received: 23.09.2018, accepted: 27.11.2018. 
activation of CD8+ T [10]. Tziotzios et al. identified four circulating microRNAs as being highly predictive of the disease status in FFA [11]. Harries et al. hypothesized that the increased scalp sweating seen in some FFA patients could be in part related to the neurogenic inflammatory process occurring locally within the skin [12]. A possible role for peroxisome proliferator activated receptor- $\gamma$ (PPAR- $\gamma$ ) in pathogenesis of LPP was suggested and remains to be elucidated in FFA [13].

The steeply rising incidence of the FFA strongly points towards environmental factors in disease aetiology. Relationship between the use of sunscreens and facial skincare products and FFA was suggested on the basis of the questionnaire studies in female and male patients [14, 15]. It should be noted that FFA was first described in Australia, where sunscreens are widely used for many years and where a recently reported FFA case might suggest that avoidance of sunscreen use on the forehead can improve disease [16]. Moreover, the same authors suggest that the presence of UV filters in hair-care products could explain the progressive pattern of FFA as well as LPP [17]. Contact allergy to hair dye and a higher concentration of hair dye products on the anterior hairline with the initial stroke of application, with reducing gradient posteriorly could explain the preferential involvement of the anterior hair margin in FFA [18]. The increased fragrance allergy and photosensitivity complaint were also found, although the authors did not confirm the association of FFA with photoallergy to several cosmetic-related substances [14, 19, 20]. Brunet-Possenti et al. suggested that the reasons for the high frequency of positive patch tests may be an extensive use of cosmetics in this group, or an altered immune response. Given the widespread use of titanium dioxide nanoparticles in leaveon cosmetics, and particularly in sunscreens, and the recent publications reporting a possible association between the use of sunscreens and the development of FFA, the authors hypothesized that a T-cell-mediated allergic response to $\mathrm{TiO}_{2} \mathrm{NP}$, as occurs to metals involved in some cases of oral lichenoid lesions, might be important in the pathophysiology of FFA [21]. The role of dietary factors in FFA was studied by Rudnicka and Rakowska and there were statistically significant differences between FFA patients and healthy individuals in regard to consumption of buckwheat and millet groats at least twice weekly in the recent 3 months $(81.4 \%$ and $30.5 \%$, respectively for the 59 FFA patients, and $22.0 \%$ and $8.4 \%$, respectively for the 59 healthy controls, $p<0.01$ ) [22]. Surprisingly, in one case series smoking was associated with less severe FFA [23]. Finally, additional findings may clarify whether the actual proposed mechanisms may play a role in FFA pathogenesis.

\section{Clinical manifestations of classical FFA}

FFA is characterized by a band of scarring alopecia localized in the frontotemporal hairline, within which perifollicular erythema and fine scale are present in the active phase of the disease (Figure 1). The lonely hair sign, a single or a few terminal hair follicles (not grouped in tufts) on the forehead standing remotely from the receding hairline, is a helpful clinical tool for diagnosing FFA. Identification of photodamage and cocking or lifting the eyebrows for identifying the location of the original hairline could be useful in clinical measurements and follow-up evaluations. Progression of the receding hairline is variable and ranges between 0.6 and $1.1 \mathrm{~cm}$ per year [24]. Frontal recession, even with longstanding FFA, is on average 1.8 to $2.6 \mathrm{~cm}$ [25]. In some patients, the parietal, occipital (7-15\%) and/or supra- and retroauricular regions are also affected. The majority (80\%) of patients experience thinning or incomplete or complete loss of eyebrows, more rarely (14\%) also of the eyelashes [26]. Other parts of the body may likewise be affected by hair loss. Near total alopecia of the arms and legs occurs commonly and often well in advance of scalp findings in FFA patients [27]. The axillae were affected in 15\% and the pubic region in $12 \%$ of cases [1]. These findings may be reported less frequently in the literature due to the delayed onset of scalp hair loss and the positive or neutral emotional reaction to hair loss in these areas. Among men, involvement of the sideburns and beard area has also been described. Loss of body hair are more often observed in men but they seem to have a lower incidence of eyebrow involvement [28]. A range of facial lesions have been reported in patients with FFA consisting of non-inflammatory, skin-coloured papules, perifollicular or diffuse erythema, glabellar red dots, depression of the frontal veins and large visible temple veins. Vellus hair follicle involvement by lichenoid inflammation, or hypertrophic sebaceous glands, and skin atrophy may justify these findings [29]. As they are subtle, their recognition may be very subjective. Hypopigmentation is commonly seen in the FFA-affected area and can be appreciated on examination with a Wood's lamp. Hyperpigmentation seems to be a unique feature of FFA seen in darker skin types and is due to lichen planus pigmentosus (LPPigm) [30].

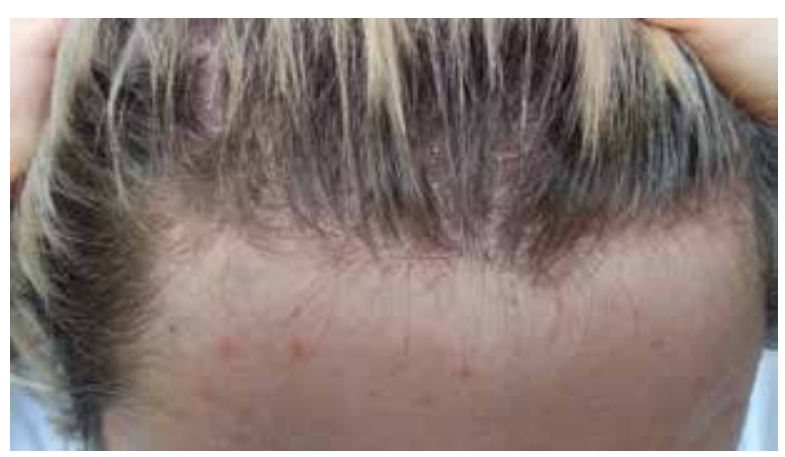

Figure 1. Frontal fibrosing alopecia: a classic linear pattern of hair loss 
Most patients with coexistence of FFA and LPPigm, found in the literature, were cases with facial involvement preceding FFA [31]. In the opinion of some authors, LPPigm and follicular red dots may not just be limited to facial skin [32-34]. An increased incidence of LPP (5.7-16.5\%), cutaneous or oral mucosal lichen planus (5.2\%) as well as lichen planus-type nail involvement or co-existence of FFA and Graham-Little-Piccardi Syndrome has also been reported [35, 36]. Interestingly, Pindado-Ortega et al. observed a high prevalence of rosacea (34\% of 103 females) in FFA [37]. While the disease is usually asymptomatic, patients occasionally report symptoms such as a feeling of tightness, a burning sensation, trichodynia or pruritus. Presence of symptoms seems to be more frequent in patients with FFA associated with concomitant LPP [38].

\section{Atypical clinical patterns of FFA}

In recent years, there has been an increasing number of reports suggesting that there are also atypical clinical FFA manifestations (Figures $2 \mathrm{~A}-\mathrm{E}$ ). Apart from the most common linear pattern of hair loss, the diffuse "zigzag" pattern and the pseudo "fringe-sign" pattern have been distinguished [39]. While in the classic linear pattern, a band of uniform frontal hairline recession in the absence of loss of hair density behind the hairline can be observed, patients with the diffuse "zigzag" pattern show significant loss of hair density behind the hairline with specific trichoscopic signs of FFA [39]. The pseudo "fringe sign", the peculiar sparing of the hairline present in some patients with FFA, was described by Pirmez et al. [40]. The true "fringe sign" is typical for traction alopecia (TA), in which the hairs of the implantation region are spared after traction. The latter is common in black patients and may coexist with FFA, making diagnosis and assessment more challenging. In a large series of 242 patients with FFA that was reported by MorenoArrones et al., the pseudo "fringe sign" affected $6.2 \%$ of subjects [39]. Rossi et al. identified three other unusual patterns of FFA that affected $18.4 \%$ of 98 patients with FFA [41]. They described six women who presented with the AGA-like pattern - marked and symmetric recession of frontotemporal hairlines, with a peculiar sparing of the paramedian frontal hairline. In 2 patients the authors observed a cockade-like pattern - bilateral oval patches of alopecia in the temporal regions, with peculiar sparing of a band of temporal hairlines, in addition to typical recession of the frontal hairline. Continuous involvement of the hairline from frontal to occipital regions - ophiasis-like pattern - was present in 6 women. Contin
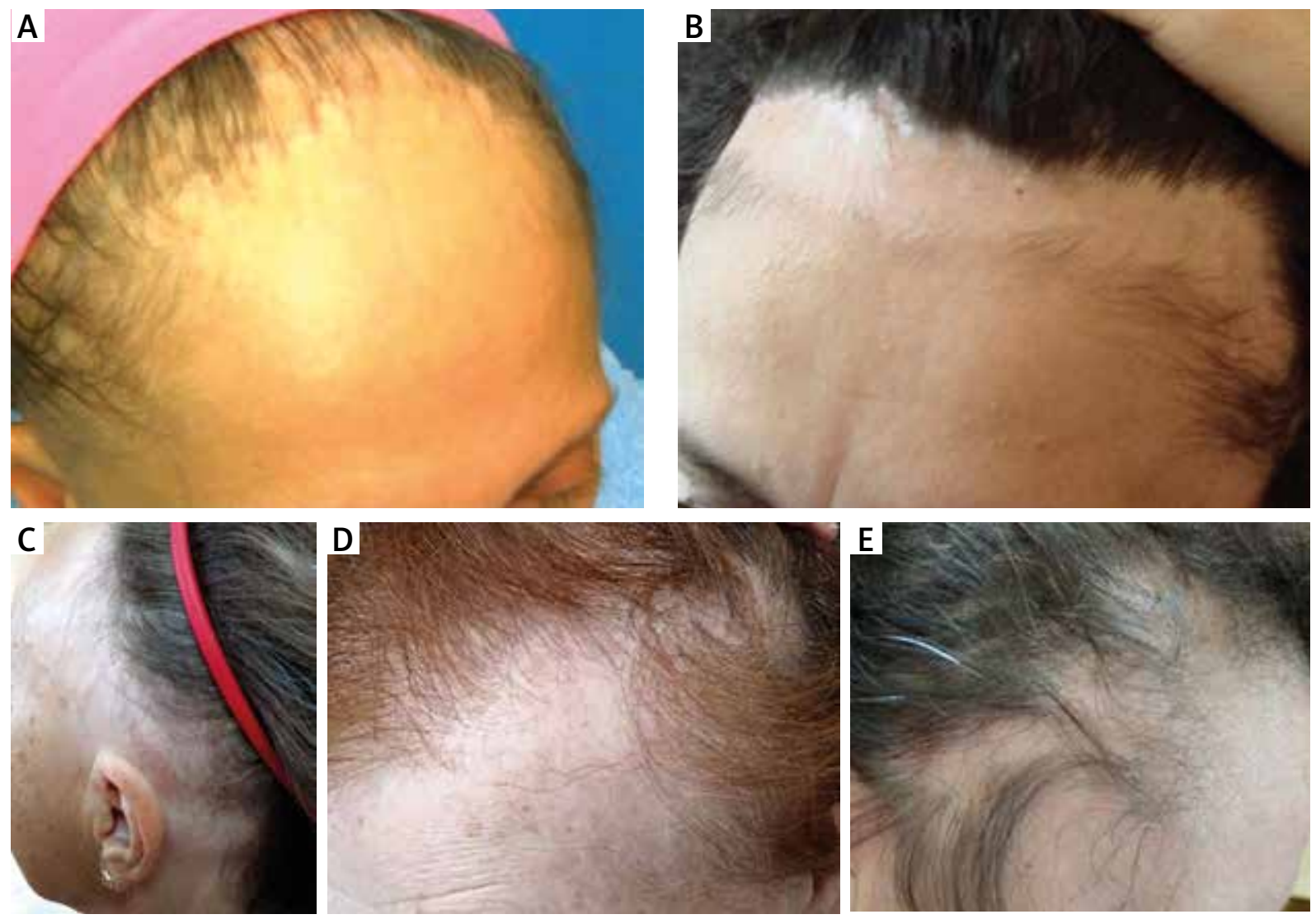

Figure 2. Atypical patterns of hair loss in frontal fibrosing alopecia: A - diffuse "zigzag", B - pseudo "fringe sign", C - ophiasis-like, D - androgenetic alopecia-like, E - cockade-like 
et al. reported six FFA cases with incomplete presentations, namely unevenly distributed irregular alopecic plaques, the presence of lonely hairs, the absence of inflammatory peripilar signs, and slow progression [42]. For that matter, on this point it should be added that FFA can be non-inflammatory on clinical presentation in locations such as the sideburns, eyebrows, trunk, and limbs. In some cases, the disease is strictly confined to the eyebrows. Loss of sideburns can be the only presenting feature in men with FFA. In such patients, the diagnosis of FFA may be overlooked.

A distinct form of cicatricial alopecia with histopathological features of LPP, termed fibrosing alopecia in a pattern distribution (FAPD), was described by Zinkernagel et al. in 2000 [43]. Katoulis et al. found the overlap of FFA, AGA and FAPD in $11 \%$ of patients [44]. Androgenetic alopecia was a common association in their patients with FFA, with a prevalence of 57\% [44]. In series of 31 patients with hair loss in the mid-frontal scalp Rakowska et al. identified the diffuse fibrotic pattern more common (64\%) than the androgenetic alopecia pattern (32\%) [45]. The observation of familial occurrence of FFA and FAPD underscores a nosologic relationship between the two conditions with respect to both the androgenetic background and the lichenoid inflammatory reaction pattern [46].

\section{Early diagnosis}

Pruritus and pain have been identified as common presenting symptoms in patients with early FFA [47]. They may show normal hair density, perifollicular erythema and scaling. In some cases, thinning of the lateral aspects of eyebrows or loss of hair on the arms and legs precede frontotemporal hair loss [24, 48]. Facial papules, follicular red dots, perifollicular or diffuse erythema and lichen planus pigmentosus can also be the first signs of FFA preceding the scalp hair loss by several months to years. These involvements in premenopausal women are frequent and allow to make an early diagnosis of the disease before developing a hairless band of atrophy on the frontotemporal scalp [49].

\section{Diagnostic criteria}

The differential diagnosis of FFA is broad, and diagnostic criteria can aid in identifying this entity. Tolkachjov et al. and Vano-Galvan et al. proposed a set of criteria for diagnosis of FFA based on the criteria described by Kossard et al. [50, 51]. The major criteria include:

1. Cicatricial alopecia of the frontal, temporal, or frontotemporal scalp on examination, in the absence of follicular keratotic papules on the body, and 2. Diffuse bilateral eyebrow cicatricial alopecia.
There are an additional 5 minor criteria, including: 1. Perifollicular erythema, perifollicular hyperkeratosis, or solitary hairs on physical or trichoscopic examination in a field of frontal/frontotemporal cicatricial alopecia.

2. Histopathologic features of cicatricial alopecia in the pattern of FFA or LPP on biopsy.

3. Involvement (hair loss, perifollicular erythema, or perifollicular hyperkeratosis) of additional FFA sites: occipital area, facial hair, sideburns, or body hair.

4. Noninflammatory facial papules.

5. Preceding or concurrent symptoms, such as pruritus or pain, at areas of involvement.

The diagnosis of FFA is made if a patient has either 2 major criteria or 1 major and 2 minor criteria.

\section{Comorbidities in FFA}

The analysis of comorbidities of FFA patients revealed that $15-31.4 \%$ of documented patients had thyroid disease, including Hashimoto's thyroiditis [1]. In addition, female FFA patients had a greater prevalence of other autoimmune diseases, in particular SLE, DLE, rheumatoid arthritis, and polymyositis [52]. However, no evidence for an increased association of FFA with rheumatologic autoimmune disorders (3\%) was found by Valesky [1].

\section{Diagnostics and monitoring}

The FFA diagnosis is based on a distinctive clinical picture, trichoscopy and, in case of doubt, histological examination.

Trichoscopy has been increasingly used for distinguishing features of FFA from other forms of alopecia, such as AGA. Trichoscopy findings of the frontotemporal line in patients with FFA include loss of follicular openings, peripilar casts and perifollicular erythema, absence of vellus and presence of lonely hairs (Figure 3 ). The "lonely hair" sign, which was seen in $25 \%$ of the patients, must not be regarded as pathognomonic for FFA as it also occurs in other forms of cicatricial alopecia. It does, however, enable the distinction from non-scarring forms such as TA. In contrast, trichoscopy abnormalities in AGA of the mid-frontal scalp include: more than $10 \%$ of thin hairs $(<0.03 \mathrm{~mm})$, hair shaft thickness diversity of $20 \%$ or more, more than four yellow dots in four images (70-fold magnification), lower average hair thickness in the frontal area than in the occiput, an increased number of follicular units with one hair, a decreased number of follicular units with three hairs and presence of the peripilar sign [53]. Trichoscopy increases diagnostic accuracy of eyelash and eyebrow loss in FFA patients and is a very useful tool to assess facial vellus hair follicle involvement because subtle clinical signs may go unnoticed [54]. In locations such as the sideburns, eyebrows, trunk, and limbs, trichoscopy does not reveal peripilar erythema or peripilar casts, but often shows transparent proximal hair emergence [55]. 

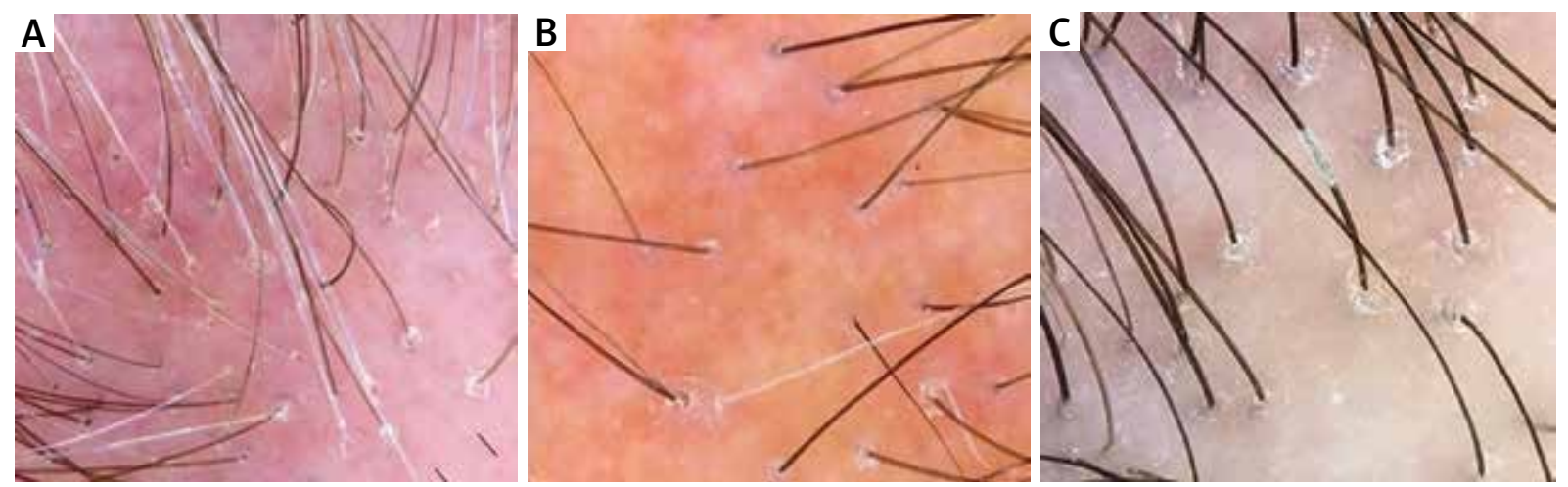

Figure 3. Trichoscopy findings in frontal fibrosing alopecia: loss of follicular openings, peripilar casts and perifollicular erythema, absence of vellus and presence of lonely hairs

Histopathology should be performed during the initial stages of the disorder in order to make a more accurate diagnosis. The absence of classic features for scarring alopecia in early cases of FFA without apparent alopecia of the hairline may have been misdiagnosed as AGA [56]. FFA-like cutaneous lupus erythematosus cases as well as association of FFA and alopecia areata in some patients were also described $[57,58]$. The FFA lesions are characterized by a lymphocytic infiltrate, perifollicular fibrosis and loss of follicles. Terminal, intermediate and vellus hairs may be affected, often termed the "follicular triad". A dense lichenoid inflammatory infiltrate around the isthmus and infundibular regions of the hair follicles whim does not extend into the hair bulb is a helpful clue to the diagnosis of early FFA. A comparative analysis of immunopathological features did not show any significant differences in direct immunofluorescence and immunohistochemistry which would allow a histological distinction to be made between FFA and LPP [59]. Evaluation of inflammatory cell types in and around hair follicles in FFA revealed a CD8-biased T-cell infiltrate with increased numbers of Langerhans cells in the infundibuloisthmic region [60]. According to Pastuszczak et al., the pre-treatment number of Langerhans cells may be a useful marker of proper response to hydroxychloroquine treatment in patients with FFA. While in patients with disease stabilization after hydroxychloroquine treatment, the pre-treatment number of CD1+ cells (Langerhans cells) within the bulge infiltration was more than $12 \%$, in those with disease progression, the pre-treatment number of CD1+ cells did not exceed 5\% [61].

Histopathology is the gold-standard for FFA diagnosis but not ideal for monitoring given the invasive nature. What is more, findings from a small skin sample may not be representative of inflammation globally. Clinical evaluation is usually used in the severity and activity assessment. As FFA is a slowly progressive disease, the pull test is negative in nearly all patients. A few scoring methods have been proposed. The scale of Vano Galvan et al. classifies FFA severity into 5 grades by measuring the area of cicatricial skin in the frontotemporal hairline. The Frontal Fibrosing Alopecia Severity Index (FFASI) score developed by Holmes et al. comprises measurement of hairline alopecia and notes the presence of hair loss elsewhere (face and body), plus other disease features including scalp LPP and facial papules [62]. The Frontal Fibrosing the Alopecia Severity Score (FFASS) proposed by Saceda-Corralo et al. is based on the evaluation of severity and extent of frontal and temporal hairline recession, eyebrow loss, perifollicular erythema and hyperkeratosis, pruritus and pain associated with FFA [63].

Trichoscopy is strongly recommended for clinical evaluation as it can detect perifollicular inflammation with higher sensibility. However, the chronic use of topical corticosteroids increases the presence of arborizing vessels and diffuse erythema. These changes noted on trichoscopy can make the correct assessment of subclinical inflammation difficult [64]. Lis-Święty et al. revealed that over $40 \%$ of patients diagnosed by trichoscopy as being in the active phase of FFA did not have such diagnosis proven histologically [65]. Therefore, the authors explored the value of trichoscopy and thermal imaging for a detection of inflammation in FFA and found that the accuracy of infrared thermography was superior to that of dermoscopy. To monitor the activity of the disease, the optical coherence tomography was also studied. It demonstrated that as disease advances from inflammatory to cicatricial, there is a progressive decrease in the vascular density of the superficial plexus and a progressive increase in vascularization of the deeper plexus [66].

Physicians should be aware of the potential association between FFA and other autoimmune diseases and should obtain haematological and biochemical blood tests, thyroid function tests, and antinuclear antibodies once the diagnosis of FFA has been established. It has been also recommended to screen for androgen excess or deficiency (total testosterone, free testosterone, free testosterone percentage, dehydroepiandrosterone sulfate, and androstenedione) in patients who are over- 
weight or obese, have polycystic ovary syndrome, ovarian cysts, or clinical evidence of elevated androgens e.g. hirsutism [67]. Patch testing should be considered in FFA patients because of the increased fragrance allergy.

\section{Prognostic factors}

Although FFA seems to be self-limiting in most cases, nonetheless the degree of progression before stabilization is not easy to predict. According to Moreno-Arrones et al. and Pirmez et al., patients with the pseudo "fringe sign" had the best prognosis $[39,68]$. They presented with less hairline recession and eyebrow involvement. Signs of disease activity and other clinical features were also less commonly present. The majority of patients remained stable with treatment. In a multicentre review of 355 patients performed by Vano-Galvan et al., initial eyebrow loss had favourable prognostic implications, while the diffuse "zigzag" pattern of hair loss, eyelash and body hair loss and facial papules were poor prognostic indicators [69]. Strazzulla et al. revealed that younger age of onset was significantly associated with a less severe disease course [70].

\section{Treatment}

Most FFA patients are treated with high-potency steroids delivered topically or intralesionally and topical calcineurin inhibitors to avoid progression of the disorder. In a German analysis of 72 patients, more than half of the patients receiving a combination of steroids and pimecrolimus subjectively reported improvement or stabilization of the condition, indicating a high degree of patient satisfaction [2]. However, the disease stabilization was observed beginning from month 9 to 12 after the onset of the tandem therapy [2]. Where AGA represents a comorbid condition, topical minoxidil, and oral $5 \alpha$-reductase inhibitors (finasteride or dutasteride) may be of benefit. However, their use should be considered in patients with a high disease burden and rapid disease progression, bearing in mind possible adverse effects. Common adverse effects of $5 \alpha$-reductase inhibitors in women include depression, headache, nausea and hot flashes. These agents are teratogenic and should be used with caution in premenopausal women $[39,71]$. According to Rakowska et al., the 12-14 months' administration of systemic retinoids (isotretinoin or acitretin) may be more beneficial [72]. The stabilization of frontal hairline was achieved in over $70 \%$ of patients treated with retinoids and in $43 \%$ of patients treated with finasteride [72]. Oral isotretinoin may also be a therapeutic option for facial papules [73-75]. Hormone replacement therapy has not shown a protective effect or efficacy as treatment [71]. Other treatment modalities include the oral administration of prednisone, antimalarial (hydroxychloroquine or chloroquine), cyclosporine, mycophenolate mofetil, azathioprine, methotrexate, thalidomide, tetracycline, spironolactone, and griseofulvin. Sadly, they have been reported in a small case series only, with varied responses. Strazzulla et al. found that patients treated with doxycycline, tetracycline, or minocycline were significantly more likely to experience adverse medication effects compared with other treatments [70]. Novel treatments are hair grafting, laser therapy, rituximab and pioglitazone [75]. It seems that the two latter drugs might be a good candidate for future studies on the treatment of FFA. According to Fertig and Tosti, excimer laser may be very effective in reducing inflammation and peripilar casts in patients with active disease [76]. Hair transplantation should only be performed in end-stage alopecic areas and in patients with no disease activity, such as itching and burning sensations, follicular hyperkeratosis, or perifollicular erythema, for at least 2 years. Close postoperative follow-ups every $4-6$ months are needed, and if disease reactivation is noted after the surgery, medical treatments should be given to prevent further follicular damage [77].

\section{Conclusions}

Currently, there is no evidence-based therapy for FFA. The primary treatment goal is to establish the diagnosis in the early stage and to prevent further progression of hair loss. Regrowth of hair is achieved only in isolated cases. However, patients may present without classic symptoms and with atypical or incomplete disease. Therefore, the diagnosis of early FFA may be overlooked.

\section{Conflict of interest}

The authors declare no conflict of interest.

\section{References}

1. Valesky EM, Maier MD, Kippenberger S, et al. Frontal fibrosing alopecia - review of recent case reports and case series in PubMed. J Dtsch Dermatol Ges 2018; 16: 992-9.

2. Heppt MV, Letulé V, Laniauskaite I, et al. Frontal fibrosing alopecia: a retrospective analysis of 72 patients from a German academic center. Facial Plast Surg 2018; 34: 88-94.

3. Atarguine $\mathrm{H}$, Hocar O, Hamdaoui A, et al. Frontal fibrosing alopecia: report on three pediatric cases. Arch Pediatr 2016; 23: 832-5.

4. Alegre-Sánchez A, Saceda-Corralo D, Bernárdez C, et al. Frontal fibrosing alopecia in male patients: a report of 12 cases. J Eur Acad Dermatol Venereol 2017; 31: e112-4.

5. Tziotzios C, Stafano CM, fenton DA, et al. Frontal fibrosing alopecia: reflections and hypotheses on aetiology and pathogenesis. Exp. Dermatol 2016; 25: 847-52.

6. Bernárdez C, Molina-Ruiz AM, Vańó-Galvan S, et al. Sex hormone status in premenopausal women with frontal fibrosing alopecia: a multicentre review of 43 patients. Clin Exp Dermatol 2017; 42: 921-3.

7. Ranasinghe GC, Piliang MP, Bergfeld WF. Prevalence of hormonal and endocrine dysfunction in patients with lichen 
planopilaris (LPP): a retrospective data analysis of $168 \mathrm{pa}-$ tients. J Am Acad Dermatol 2017; 76: 314-20.

8. Lobato-Berezo A, March-Rodríguez A, Deza G, et al. Frontal fibrosing alopecia after antiandrogen hormonal therapy in a male patient. J Eur Acad Dermatol Venereol 2018; 32: e291-2.

9. Katoulis AC, Diamanti K, Sgouros D, et al. Frontal fibrosing alopecia: is the melanocyte of the upper hair follicle the antigenic target? Int J Dermatol 2018; 57: e37-8.

10. Lin J, Valdebran M, Bergfeld W, et al. Hypopigmentation in frontal fibrosing alopecia. J Am Acad Dermatol 2017; 76: 1184-6.

11. Tziotzios C, Ainali C, Holmes S. Tissue and circulating microRNA co-expression analysis shows potential involvement of miRNAs in the pathobiology of frontal fibrosing alopecia. J Invest Dermatol 2017; 137: 2440-3.

12. Harries MJ, Wong S, Farrant P. Frontal fibrosing alopecia and increased scalp sweating: is neurogenic inflammation the common link? Skin Appendage Disord 2016; 1: 179-84.

13. Harries MJ, Jimenez F, Izeta A, et al. Lichen planopilaris and frontal fibrosing alopecia as model epithelial stem cell diseases. Trends Mol Med 2018; 24: 435-48.

14. Aldoori N, Dobson K, Holden CR. Frontal fibrosing alopecia: possible association with leave on facial skin care products and sunscreens; a questionnaire study. Br J Dermatol 2016; 175: 762-7.

15. Debroy-Kidambi A, Dobson K, Holmes S, et al. Frontal fibrosing alopecia in men: an association with facial moisturizers and sun-screens. Br J Dermatol 2017; 177: 260-1.

16. Cranwell WC, Sinclair R. Frontal fibrosing alopecia: regrowth following cessation of sunscreen on the forehead. Australas J Dermatol 2019; 60: 60-1.

17. Callander J, Frost J, Stone N. Ultraviolet filters in hair-care products: a possible link with frontal fibrosing alopecia and lichen planopilaris. Clin Exp Dermatol 2018; 43: 69-70.

18. Kumaran MS, Razmi TM, Vinay K, Parsad D. Clinical, dermoscopic, and trichoscopic analysis of frontal fibrosing alopecia associated with acquired dermal macular hyperpigmentation: a cross sectional observational case-control study. J Am Acad Dermatol 2018; 79: 588-91.

19. Donati A. Frontal fibrosing alopecia and sunscreens: cause or consequence? Br J Dermatol 2016; 175: 675-6.

20. Rocha VB, Donati A, Contin LA. Photopatch and patch testing in 63 frontal fibrosing alopecia patients: a case series. Br J Dermatol 2018; 179: 1402-3.

21. Brunet-Possenti F, Deschamps L, Colboc H, et al. Detection of titanium nanoparticles in the hair shafts of a patient with frontal fibrosing alopecia. J Eur Acad Dermatol Venereol 2018; 32: e442-3.

22. Rudnicka L, Rakowska A. The increasing incidence of fronta fibrosing alopecia. In search of triggering factors. J Eur Acad Dermatol Venereol 2017; 31: 1579-80.

23. Fonda-Pascual P, Saceda-Corralo D, Moreno-Arrones OM, et al. Frontal fibrosing alopecia and environment: may tobacco be protective? J Eur Acad Dermatol Venereol 2017; 31: e98-9.

24. To D, Beecker J. Frontal fibrosing alopecia: update and review of challenges and successes. J Cutan Med Surg 2018; 22: 182-9.

25. Vano-Galvan S, Molina-Ruiz AM, Serrano-Falcon C, et al. Frontal fibrosing alopecia: a multicenter review of $355 \mathrm{pa}$ tients. J Am Acad Dermatol 2014; 70: 670-8.

26. Tolkachjov SN, Chaudhry HM, Camilleri MJ, Torgerson RR. Frontal fibrosing alopecia among men: a clinicopathologic study of 7 cases. J Am Acad Dermatol 2017; 77: 683-90.e2.
27. Dina Y, Okoye GA, Aguh C. The timing and distribution of non-scalp hair loss in patients with lichen planopilaris and frontal fibrosing alopecia: a survey-based study. J Am Acad Dermatol 2018; 17: pii: S0190-9622(18)30464-X.

28. Ormaechea-Pérez N, López-Pestańa A, Zubizarreta-Salvador J, et al. Frontal fibrosing alopecia in men: presentations in 12 cases and a review of the literature. Actas Dermosifiliogr 2016; 107: 836-44.

29. Pedrosa AF, Duarte AF, Haneke E, Correia O. Yellow facial papules associated with frontal fibrosing alopecia: a distinct histologic pattern and response to isotretinoin. J Am Acad Dermatol 2017; 77: 764-6.

30. Petrof G, Cuell A, Rajkomar VV, et al. Retrospective review of 18 British South Asian women with frontal fibrosing alopecia. Int J Dermatol 2018; 57: 490-1.

31. Mulinari-Brenner FA, Guilherme MR, Peretti MC, Werner B. Frontal fibrosing alopecia and lichen planus pigmentosus: diagnosis and therapeutic challenge. An Bras Dermatol 2017; 92: 79-81.

32. Pirmez R, Donati A, Valente NS, et al. Glabellar red dots in frontal fibrosing alopecia: a further clinical sign of vellus follicle involvement. Br J Dermatol 2014; 170: 745-6.

33. Franco-Muńoz M, García-Arpa M, Lozano-Masdemont B, Ramos-Rodríguez C. Frontal fibrosing alopecia and extrafacial lichen planus pigmentosum in a caucasian woman. An Bras Dermatol 2017; 92: 76-8.

34. Meyer V, Sachse M, Rose C, Wagner G. Follicular red dots of the hip in frontal fibrosing alopecia - do we have to look twice? J Dtsch Dermatol Ges 2017; 15: 327-8.

35. Nascimento LLD, Enokihara MM, Vasconcellos MRA. Coexistence of chronic cutaneous lupus erythematosus and frontal fibrosing alopecia. An Bras Dermatol 2018; 93: 274-6.

36. Macpherson M, Hohendorf-Ansari P, Trüeb RM. Nail involvement in frontal fibrosing alopecia. Int J Trichology 2015; 7 : 64-6.

37. Pindado-Ortega C, Saceda-Corralo D, Buendía-Castańo D, et al. Frontal fibrosing alopecia and cutaneous comorbidities: a potential relationship with rosacea. J Am Acad Dermatol 2018; 78: 596-7.e1.

38. Saceda-Corralo D, Fernández-Crehuet P, Fonda-Pascual P, et al. Clinical description of frontal fibrosing alopecia with concomitant lichen planopilaris. Skin Appendage Disord 2018; 4: 105-7.

39. Moreno-Arrones OM, Saceda-Corralo D, Fonda-Pascual P, et al. Frontal fibrosing alopecia: clinical and prognostic classification. J Eur Acad Dermatol Venereol 2017; 31: 1739-45.

40. Pirmez R, Duque-Estrada B, Abraham LS, et al. It's not all traction: the pseudo 'fringe sign' in frontal fibrosing alopecia. Br J Dermatol 2015; 173: 1336-8.

41. Rossi A, Grassi S, Fortuna MC, et al. Unusual patterns of presentation of frontal fibrosing alopecia: a clinical and trichoscopic analysis of 98 patients. J Am Acad Dermatol 2017; 77: 172-4.

42. Contin LA, de Almeida Ledá YL, Caldeira Nassif K, Suárez Restrepo MV. Patchy frontal fibrosing alopecia: description of an incomplete clinical presentation. Skin Appendage Disord 2017; 3: 190-2.

43. Zinkernagel MS, Trüeb RM. Fibrosing alopecia in a pattern distribution: patterned lichen planopilaris or androgenetic alopecia with a lichenoid tissue reaction pattern? Arch Dermatol 2000; 136: 205-11.

44. Katoulis AC, Diamanti K, Sgouros D, et al. Is there a pathogenetic link between frontal fibrosing alopecia, androgenetic 
alopecia and fibrosing alopecia in a pattern distribution? J Eur Acad Dermatol Venereol 2018; 32: e218-20.

45. Rakowska A, Waśkiel A, Sikora M, et al. Two different trichoscopic patterns of mid-frontal scalp in patients with frontal fibrosing alopecia and clinical features of androgenetic alopecia. Dermatol Rev 2017; 104: 9-15.

46. Missio DM, Dias MF, Trüeb RM. Familial cicatricial alopecia: report of familial frontal fibrosing alopecia and fibrosing alopecia in a pattern distribution. Int J Trichology 2017; 9: 130-4.

47. Banka N, Mubki T, Bunagan MJ, et al. Frontal fibrosing alopecia: a retrospective clinical review of 62 patients with treatment outcome and long-term follow-up. Int J Dermat 2014; 53: 1324-30.

48. Anzai A, Donati A, Valente NY, et al. Isolated eyebrow loss in frontal fibrosing alopecia: relevance of early diagnosis and treatment. Br J Dermatol 2016; 175: 1099-101.

49. Romiti R, Biancardi Gavioli CF, Anzai A, et al. Clinical and histopathological findings of frontal fibrosing alopecia-associated lichen planus pigmentosus. Skin Appendage Disord 2017; 3: 59-63.

50. Tolkachjov SN, Chaudhry HM, Imhof RL, et al. Reply to: "Updated diagnostic criteria for frontal fibrosing alopecia". J Am Acad Dermatol 2018; 78: e23-4.

51. Vańó-Galván S, Saceda-Corralo D, Moreno-Arrones OM, et al. Updated diagnostic criteria for frontal fibrosing alopecia. J Am Acad Dermatol 2018; 78: e21-2.

52. Fertig RM, Hu S, Maddy AJ, et al. Medical comorbidities in patients with lichen planopilaris, a retrospective case-control study. Int I Dermatol 2018; 57: 804-9.

53. Rakowska A, Olszewska M, Rudnicka L. Frontal fibrosing alopecia. In: Atlas of Trichoscopy. Rudnicka L, Olszewska M, Rakowska A. (eds.) Springer-Verlag, London 2012; 295-301.

54. López-Pestańa A, Tuneu A, Lobo C, et al. Facial lesions in frontal fibrosing alopecia (FFA): clinicopathological features in a series of 12 cases. J Am Acad Dermatol 2015; 73: 987. e1-6.

55. Cervantes J, Miteva M. Distinct trichoscopic features of the sideburns in frontal fibrosing alopecia compared to the frontotemporal scalp. Skin Appendage Disord 2018; 4: 50-4.

56. Miteva M, Sabiq S. A new histologic pattern in 6 biopsies from early frontal fibrosing alopecia. Am J Dermatopathol 2019; 41: 118-121.

57. Lin J, Zikry J, Atanaskova-Mesinkovska N. Development of frontal fibrosing alopecia with a history of alopecia areata. Int J Trichology 2018; 10: 29-30.

58. Trüeb RM, El Shabrawi-Caelen L, Kempf W. Cutaneous lupus erythematosus presenting as frontal fibrosing alopecia: report of 2 patients. Skin Appendage Disord 2017; 3: 205-10.

59. Gálvez-Canseco A, Sperling L. Lichen planopilaris and frontal fibrosing alopecia cannot be differentiated by histopathology. J Cutan Pathol 2018; 45: 313-7.

60. Ma SA, Imadojemu S, Beer K, Seykora JT. Inflammatory features of frontal fibrosing alopecia. J Cutan Pathol 2017; 44: 672-6.

61. Pastuszczak M, Wawrzynkiewicz M, Dyduch G, Wojas-Pelc A. Langerhans cells as a histologic marker of proper treatment response to hydroxychloroquine in patients with frontal fibrosing alopecia. $1^{\text {st }}$ World Congress of Trichoscopy "All about hair \& nails", Warsaw [Poland] 15-17.03.2018, abstracts. Dermatol Rev 2018; 105: 136.

62. Holmes S, Ryan T, Young D et al. Frontal Fibrosing Alopecia Severity Index (FFASI): a validated scoring system for assessing frontal fibrosing alopecia. Br I Dermatol 2016; 175: 203-7.
63. Saceda-Corralo D, Moreno-Arrones OM, Pindado-Ortega C, Vańo-Galván S. Development and validation of the frontal fibrosing alopecia severity score: response from authors. J Am Acad Dermatol 2018; 78: 522-9.

64. Saceda-Corralo D, Moreno-Arrones OM, Fonda-Pascual P, et al. Steroid-induced changes noted on trichoscopy of patients with frontal fibrosing alopecia. J Am Acad Dermatol 2018; 79: 956-7.

65. Lis-Święty A, Miziołek B, Ranosz-Janicka I, et al. Thermal imaging and dermoscopy for detecting inflammation in frontal fibrosing alopecia. J Cosmet Dermatol 2018; 17: 268-73.

66. Vazquez-Herrera NE, Eber AE, Martinez-Velasco MA, et al. Optical coherence tomography for the investigation of frontal fibrosing alopecia. J Eur Acad Dermatol Venereol 2018; 32: $318-22$.

67. Ranasinghe GC, Piliang MP, Bergfeld WF. Prevalence of hormonal and endocrine dysfunction in patients with lichen planopilaris (LPP): a retrospective data analysis of $168 \mathrm{pa}$ tients. J Am Acad Dermatol 2017; 76: 314-20.

68. Pirmez R, Vańó-Galván S. Acknowledging the pseudo "fringe sign" in frontal fibrosing alopecia has diagnostic and prognostic implications. J Am Acad Dermatol 2018; 78: e19.

69. Vano-Galvan S, Molina-Ruiz AM, Serrano-Falcon C, et al. Frontal fibrosing alopecia: a multicenter review of $355 \mathrm{pa}$ tients. J Am Acad Dermatol 2014; 70: 670-8.

70. Strazzulla LC, Avila L, Li X, et al. Prognosis, treatment, and disease outcomes in frontal fibrosing alopecia: a retrospective review of 92 cases. J Am Acad Dermatol 2018; 78: 203-5.

71. Rácz E, Gho C, Moorman PW, et al. Treatment of frontal fibrosing alopecia and lichen planopilaris: a systematic review. J Eur Acad Dermatology Venereol 2013; 27: 1461-70.

72. Rakowska A, Gradzińska A, Olszewska M, Rudnicka L. Efficacy of isotretinoin and acitretin in treatment of frontal fibrosing alopecia: retrospective analysis of 54 cases. J Drugs Dermatol 2017; 16: 988-92.

73. Flores-Terry MÁ, García-Arpa M, Franco-Muńóz M, GonzálezRuiz L. Facial papules in frontal fibrosing alopecia: good response to isotretinoin. Actas Dermosifiliogr 2018; 109: 831-3.

74. Pirmez R, Barreto T, Duque-Estrada B, et al. Successful treatment of facial papules in frontal fibrosing alopecia with oral isotretinoin. Skin Appendage Disord 2017; 3: 111-3.

75. Bomar L, McMichael 1. Frontal fibrosing alopecia. Br J Dermatol 2017; 177: e58-9.

76. Fertig R, Tosti A. Frontal fibrosing alopecia treatment options. Intractable Rare Dis Res 2016; 5: 314-5.

77. Liu YS, Jee SH, Chan JL. Hair transplantation for the treatment of lichen planopilaris and frontal fibrosing alopecia: a report of two cases. Australas J Dermatol 2018; 59: e118-22. 